\title{
Factores Asociados a la Repetición de Cursos y Retraso en la Graduación en Programas de Ingeniería de la Universidad de Cartagena, en Colombia
}

\author{
Diofanor Acevedo ${ }^{(1)}$ *, José D. Torres ${ }^{(1)}$ y Moisés J. Jiménez ${ }^{(2)}$ \\ (1) Universidad de Cartagena, Facultad de Ingeniería, Programa de Ingeniería de Alimentos, Avenida el \\ Consulado, Calle 30 No. 48-152. Cartagena, Bolívar-Colombia (e-mail:diofanor3000@gmail.com) \\ (2) Universidad Antonio Nariño, Facultad de Ingeniería Electrónica y Biomédica, Avenida Crisanto Luque, \\ Dg. 22 No. 48 A - 64. Cartagena, Bolívar-Colombia
}

* Autor a quien debe ser dirigida la correspondencia

Recibido Sep. 3, 2014; Aceptado Nov. 19, 2014; Versión final recibida Ene. 11, 2015

\begin{abstract}
Resumen
En la presente investigación se identifican y discuten los factores asociados a la repetición de cursos y retraso en la graduación en los programas de Ingeniería de la Universidad de Cartagena, en Colombia. Diversas investigaciones afirman que la repetición y el retraso académico son indicadores de deficiencias de los sistemas educativos, cuestión que es analizada en este trabajo. Se revisaron boletines estadísticos de la Universidad de Cartagena, así como de los Sistemas de información del Ministerio de Educación de Colombia. Los mayores porcentajes de repetición se estuvieron en el programa Ingeniería de Alimentos $(31.4 \%)$ y en el área de Física (33.9\%). No se detectaron diferencias entre programas $(p>0.05)$.De los resultados se evidencia que la repetición fue debida a malos hábitos de estudio, lo cual no varió por géneros $(p>0.05)$. El retraso académico se asoció a no haber terminado el trabajo de término de carrera (tesis de grado), lo que fue distinto entre géneros $(\mathrm{p}<0.05)$.
\end{abstract}

Palabras clave: programas de ingeniería, repetición de cursos, retraso académico, sistema educacional

\section{Factors Associated With Course Repetition and Graduation Delay in Engineering Programs of the University of Cartagena in Colombia}

\begin{abstract}
In the present investigation the factors associated with course repetition and delay of graduation in the Engineering programs of the University of Cartagena in Colombia, were identified and discussed. Several studies suggest that repetition and academic delay are indicative of failure of the education systems, aspects that are analyzed in this work. Statistical bulletins of the University of Cartagena and data from the Information Systems of the Colombian Ministry of Education were reviewed. The highest percentages of repetition were found in the Food Engineering program (31.4\%) and in the area of physics (33.9\%). No differences between programs ( $p>0.05)$ were detected. It is evident, from the results, that repetition was due to poor study habits, which did not vary by gender $(p>0.05)$. The academic delay was associated with delay in the graduation work (bachelor thesis) which was different between genders $(p<0.05)$.
\end{abstract}

Keywords: engineering programs, course repetition, academic delay, graduation delay, educational system 


\section{INTRODUCCIÓN}

La repetición se entiende como el hecho de cursar reiterativamente una actividad académica en un período determinado (año, semestre o trimestre), esta se refleja en el retraso académico, que se refiere a la cantidad de tiempo por sobre lo establecido, que tarda un estudiante en culminar sus estudios y obtener el título que lo acredita como profesional (Carvajal et al., 2011; Baquerizo et al., 2014). Actualmente existe una preocupación creciente acerca de la magnitud y la gravedad que implican estos fenómenos, especialmente en ámbitos universitarios tales como las Facultades de Ingenierías (Quintero et al., 2010; Barragán, 2011). La repetición académica en las Universidades se ve influenciada por el nivel de preparación de los docentes, su interrelación con los alumnos, sus experiencias, los métodos de enseñanza usados, también por la forma de organización de las instituciones, su infraestructura, su modelo de gestión, la dotación de recursos didácticos disponibles para el aprendizaje y su nivel de adaptación a las características culturales, económicas y lingüísticas de los alumnos (Santamaría y Bustos, 2013; Baquerizo et al., 2014).

Diversas investigaciones afirman que la repetición y el retraso académico son indicadores de deficiencias de los sistemas educativos, ya que se ven doblegados los esfuerzos de los gobiernos en cuanto a las inversiones por alumno en cada periodo lectivo, así como también las consecuencias que trae a nivel personal y socioeconómico para quienes caen en estos fenómenos (González, 2005). Los repitentes en los primeros semestres ocupan lugares que podrían estar disponibles para los demás alumnos que requieren un cupo y que deberían matricularse, generándose así un aprovechamiento inadecuado de los espacios (Garbanzo-Vargas et al., 2007; Cerpa et al., 2014). Otros autores afirman que la repetición y el retraso académico en la educación superior, disminuye la autoestima, de los alumnos y por tanto afectan todo el entorno que rodea al individuo, ya que el alumno que repite o permanece mucho tiempo improductivo en la Universidad, comienza a percibirse interiormente como incapaz de afrontar con éxito la enseñanza y el proceso de desarrollo profesional (Tejada et al., 2013).

González, (2005) afirma que la autoestima es donde reside la causa principal de la repetición y que la autoconfianza es uno de los factores claves en el rendimiento académico. Algunos investigadores consideran a la repetición como una solución a los problemas de aprendizaje de los alumnos, pensando que éstos puede alcanzar un rendimiento académico mejor, si ven más tiempo los mismos contenidos curriculares (Soria-Barreto y. Zúñiga-Jara, 2014). Sin embargo otras investigaciones indican que ésta tiene escasa eficiencia pedagógica sobre el aprendizaje y que desde todos los puntos de vista es un gasto inútil (Rodríguez, 2013). De esta manera queda descartada la presunción de que existe conexión entre el elevado índice de repetición y la búsqueda de la excelencia académica como justificación de la misma (GarbanzoVargas et al., 2007).

Los altos índices de repetición y retraso académico van ligados a un notable incremento de los porcentaje de alumnos con edades superiores a las correspondientes para el grado o los semestres en que se matriculan (Cortes et al., 2011). La labor pedagógica se complica cuando se tiene que enseñar a alumnos con distintos niveles de madurez física y psicológica, ya que los materiales didácticos y los métodos de enseñanza están diseñados generalmente a partir de una situación ideal donde los alumnos no repitan las asignaturas vistas en un periodo académico (Tejada et al., 2013). De esta forma la repetición puede producir más repetición en lugar de mayor aprendizaje (Barragán, 2011).

En síntesis las investigaciones recopiladas en la literatura, coinciden en aseverar que la repetición y el retraso académico, son contraproducentes y poco efectivos, debido a que generan en los alumnos: frustración, bajo rendimiento, sobre-edad, deserción escolar, incremento de los problemas de aprendizaje, presión en el ambiente académico, baja autoestima, repetición de lo aprendido, costo elevado para el sistema educativo, retraso en el tiempo, e incremento innecesario en el número de estudiantes por aulas (Oliver et al., 2011; Baquerizo et al., 2014). Por ello es importante y se hace necesario el estudio de todos los factores que asociados a estos fenómenos, para de esta manera diseñar estrategias efectivas que permitan prevenirlos y solucionarlos. El objetivo de este trabajo fue identificar los factores principales asociados a la repetición y al retraso académico en la Facultad de Ingeniería de la Universidad de Cartagena.

\section{MATERIALES Y MÉTODOS}

Para el desarrollo de este estudio se empleó un diseño metodológico descriptivo, no experimental y de corte transversal, durante el segundo semestre del 2013. La netodologia se presenta en cinco sub-secciones: Población, muestra y descripción del ciclo básico de la Facultad de Ingeniería, procedimiento de recolección de información, fuentes secundarias, las fuentes primarias e instrumentos de la investigación y el análisis de los datos 


\section{Población, muestra y descripción del ciclo básico de la Facultad de Ingeniería}

La población objeto de estudio estuvo conformada por todos los alumnos matriculados en los cuatro programas, Ingeniería de Alimentos, Ingeniería Química, Ingeniería Civil e Ingeniería de Sistemas, de la Facultad de Ingeniería de la Universidad de Cartagena durante el periodo analizado, aproximadamente 1600. Se utilizó el método de muestreo no probabilístico, por ello se tomó una muestra aleatoria representativa del $10 \%$, es decir 160 alumnos distribuidos de forma equitativa por género ( $50 \%$ hombres) y ( $50 \%$ mujeres). Esta muestra fue dividida en dos subgrupos, tal como se esquematiza en la Tabla 1 . Uno con alumnos de cuarto semestre cuya edad promedio fue de 19,23 $\pm 0,75$ años, y que dentro de su hoja de vida académica presentaron una alta repetición en las asignaturas del ciclo básico. Y otro con alumnos matriculados en décimo semestre con una edad de 22,03 $\pm 0,92$ años, que iniciaron sus actividades académicas en el segundo periodo del 2009 y que debieron graduarse como ingenieros en el segundo periodo del 2013, esto con la finalidad de averiguar las causas de su retraso académico.

Tabla 1: Muestra seleccionada en los programas de la Facultad de Ingeniería Universidad de Cartagena

\begin{tabular}{|l|c|c|c|c|c|c|c|c|c|}
\hline Programas & \multicolumn{2}{|c|}{ Alimentos } & \multicolumn{2}{c|}{ Química } & \multicolumn{2}{c|}{ Sistemas } & \multicolumn{2}{c|}{ Civil } & \multirow{2}{*}{ Total } \\
\cline { 1 - 9 } Grupos/Género & Hombres & Mujeres & Hombres & Mujeres & Hombres & Mujeres & Hombres & Mujeres & \\
\hline (A) IV semestre & 10 & 10 & 10 & 10 & 10 & 10 & 10 & 10 & 80 \\
\hline (B) X semestre & 10 & 10 & 10 & 10 & 10 & 10 & 10 & 10 & 80 \\
\hline Total & 20 & 20 & 20 & 20 & 20 & 20 & 20 & 20 & 160 \\
\hline
\end{tabular}

En la Tabla 2, se detallan las asignaturas comunes en el ciclo básico de los programas de la Facultad de Ingeniería de la Universidad de Cartagena

Tabla 2: Asignaturas del ciclo básico en la Facultad de Ingeniería Universidad de Cartagena

\begin{tabular}{|l|c|l|c|}
\hline Asignaturas de I y II semestre & Créditos & Asignatura de III y IV semestre & Créditos \\
\hline Fundamentos de Matemáticas & 3 & Física II & 4 \\
\hline Cálculo Diferencial & 3 & Física III & 4 \\
\hline Química General & 4 & Cálculo Vectorial & 3 \\
\hline Cálculo Integral & 3 & Probabilidad y Estadística & 4 \\
\hline Álgebra Lineal & 3 & Ecuaciones Diferenciales & 2 \\
\hline Física I & 4 & Ecología y Medio ambiente & 1 \\
\hline
\end{tabular}

Se resalta que las asignaturas del ciclo básico, tienen el mismo número de créditos en los cuatro programas y se ubican entre los semestres de primero a cuarto. Se entiende que un crédito académico es la relación entre una hora de trabajo en el aula de clase y dos de forma independiente por parte de los alumnos. Por lo tanto las asignaturas entre mayor número de créditos tengan, más horas de dedicación al estudio deben empleárseles.

\section{Procedimiento de recolección de información}

La información requerida para la investigación fue recopilada mediante dos formas: una secundaria a través de consultas en documentos institucionales y una primaria mediante el procesamiento de los datos obtenidos de dos cuestionarios aplicados directamente a los alumnos y diseñados para averiguar las causas de la repetición y el retraso académico en la Facultad de Ingeniería de la Universidad de Cartagena.

\section{Fuentes secundarias}

Los índices de repetición y retraso académico, se obtuvieron al revisar los boletines estadísticos institucionales de la Facultad de Ingenierías de la Universidad de Cartagena, en el periodo de estudio analizado. Para contrastar los datos de repetición y retraso académico con otras Facultades en Colombia, y para obtener los datos sobre los resultados de las pruebas de estado de los programas de la Facultad de Ingeniería, se consultaron también documentos facilitados del Sistema de Aseguramiento de la Calidad de Educación Superior (SACES, 2014), y del Instituto Colombiano para el Fomento de la Educación Superior (ICFES, 2014). Para el cálculo del porcentaje promedio de repetición en las asignaturas del ciclo básico se utilizó la ecuación 1. 
$\% I . R(t)=\left(\frac{E \cdot R}{E \cdot M}\right) * 100$

Dónde: \% I.R (t) es el índice de repetición en el tiempo de referencia; E.M el número de alumnos matriculados y $\mathrm{E}$. R el número de alumnos que reprobaron

\section{Fuentes primarias e Instrumentos de la investigación}

Para investigar las principales causas de la repetición en las asignaturas del ciclo básico, al primer grupo conformado por 80 alumnos de cuarto semestre (50\% hombres y $50 \%$ mujeres), se le solicitó que diligenciara de forma presencial la parte $(A)$ del cuestionario corto diseñado para esta investigación, lo cual fue muy similar a lo realizado por González, (2005) y Oliver et al., (2011). Los alumnos se identificaron, colocando género, edad, programa, semestre y asignatura que más repitieron. Las pruebas se realizaron en las instalaciones del grupo de investigación Nutrición, Salud y Calidad Alimentaria, de la Facultad de Ingeniería de la Universidad de Cartagena. Esta parte del instrumento constó de una pregunta sencilla con once ítems como opciones de respuesta, donde cada alumno debió señalar solo la que más se acercara a la causa de su repetición. Así mismo al segundo grupo compuesto también por 80 alumnos matriculados en décimo semestre. Se le suministró pero de forma virtual la parte B del cuestionario, para indagar sobre las causas asociadas a su retraso académico, se realizó de esta manera, ya que fue difícil reunirlos a todos en forma presencial, por ello se decidió enviar dicho formato a los correos electrónicos extraídos de las hojas de vida académica de estos alumnos. Esta parte del instrumentó estuvo conformada también por una pregunta sencilla pero con diez ítems de opciones, donde se debió señalar solo una respuesta. Lo cual fue similar al utilizado por Carvajal et al., (2011).

\section{Análisis de los datos}

Después de obtener la información se construyó una base de datos en el programa EXCEL 2013 para Windows, y posteriormente en el Programa Estadístico para Ciencias Sociales, IBM SPSS @ - versión 19.0. Se introdujeron las respuestas dadas por los estudiantes en los cuestionarios, y se analizaron los correspondientes porcentajes y promedios con sus desviaciones estándar. Se realizó un análisis de varianza (ANOVA de un factor) a los porcentajes de repetición de las asignaturas de física y cálculo, entre los cuatro programas de la Facultad de Ingeniería, para hallar las diferencias estadísticamente significativas y como post hoc se aplicó la prueba de Tukey al $5 \%(p \leq 0,05)$. Así mismo, a los datos recopilados de los años 2010, 2011, 2012, y 2013, de las pruebas Saber Pro, se le realizó también un análisis de varianza para establecer si se presentaron diferencias estadísticamente significativas entre los cuatro programas.

\section{RESULTADOS Y ANÁLISIS}

En la Figura 1, se esquematizan los porcentajes de repetición en el área de física, los cuales fueron los más altos en al analizar todos los datos de las asignaturas del ciclo básico de la Facultad Ingenierías de la Universidad de Cartagena.

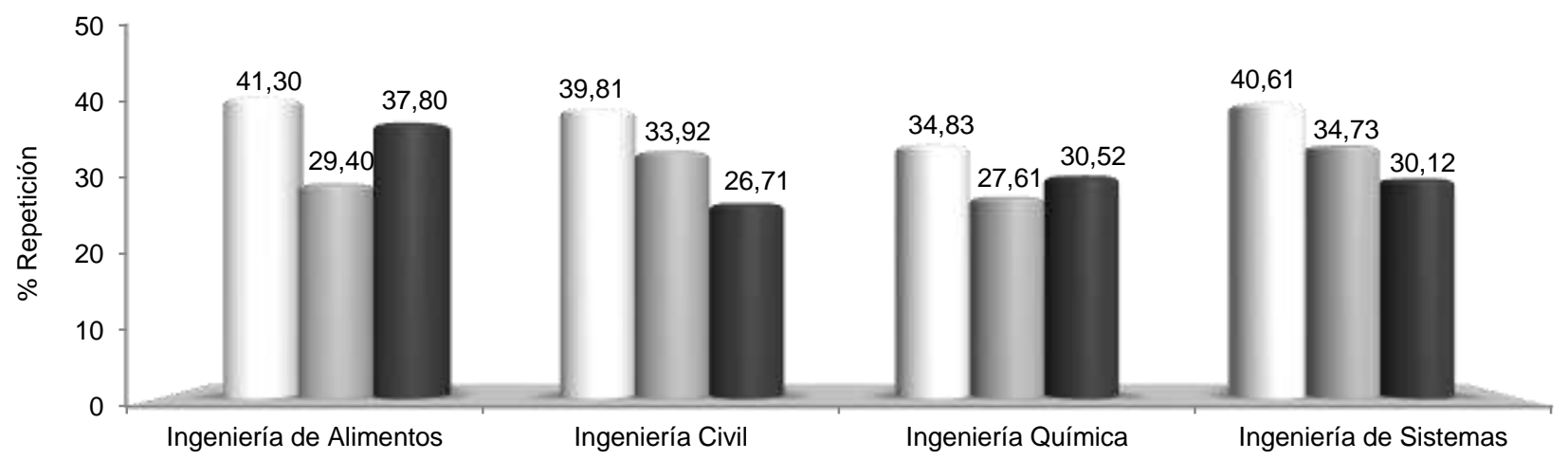

Fig. 1: Porcentaje de repetición en las áreas de Física de la Facultad de Ingeniería de la Universidad de Cartagena durante segundo periodo académico del 2013. $\square$ Física I $\square$ Física II $\mathbf{E}$ Física III

Se observa que fue física I (mecánica) con un porcentaje promedio de 39,13\%, la de mayor dificultad en todos los programas, seguida por física III (térmica) (31,40\%), física II (eléctrica) $(31,28 \%$ ). El análisis de varianza realizado a los promedios de repetición de las áreas de física (mecánica, eléctrica y térmica), no detectó diferencias estadísticamente significativas entre los cuatro programas de la Facultad de Ingeniería de la Universidad de Cartagena (Valor $F=0,51$ y $p=0,69>0,05$ ). Lo que se confirmó ya que la prueba de 
HSD de Tukey al 5\% de nivel de significancia, ubicó todos los promedios Ingeniería Química $(30,96 \pm 3,62)$, Ingeniería Civil $(33,47 \pm 6,56)$, Ingeniería de Sistemas $(35,13 \pm 5,26)$, Ingeniería de Alimentos $(36,16 \pm 6,12)$, dentro del mismo subconjunto (letras iguales).

En la Figura 2, se detallan los porcentajes de repetición de las otras asignaturas del ciclo básico. Se puede ver que las áreas de cálculo presentaron los niveles más altos, siendo en su orden el diferencial $(32,90 \%)$, el integral $(30,16 \%)$, y vectorial $(26,12 \%)$. El análisis de varianza efectuado entre los promedios de repetición en el área de cálculo, si detectó diferencias estadísticamente significativas entre los cuatro programas (Valor $F=5,14$ y $p=0,03<0,05)$.

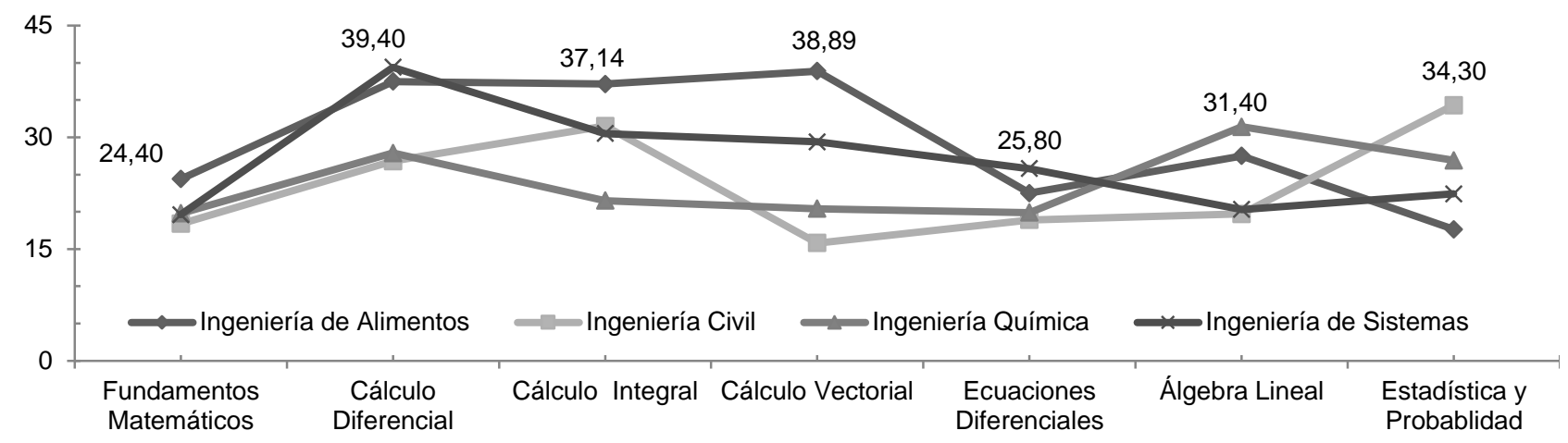

Fig. 2: Niveles de repetición académica por programas en la Facultad de Ingeniería de la Universidad de Cartagena durante segundo periodo académico del 2013.

Al aplicar la prueba de HSD de Tukey, se observó que entre Ingeniería Química $(23,27 \pm 4,05)$ e Ingeniería Civil $(24,72 \pm 6,05$ ), no hubo diferencias (letras iguales), pero entre estos dos programas, respecto a Ingeniería de Sistemas $(33,10 \pm 5,48)$, e Ingeniería de Alimentos $(37,84 \pm 0,92)$, si se presentaron diferencias. Al comparar todos los porcentajes de repetición por programa se observó que los índices más altos estuvieron en Ingeniería de Alimentos $(31,41 \%)$, e Ingeniería de Sistemas $(29,28 \%)$, seguidos por Ingeniería Civil (26,58\%) e Ingeniería Química (26,07\%).

En la Figura 3, se presenta la repetición en el área de Química en la Facultad de Ingeniería. Se observa fue de $(20,81 \%)$, siendo los programas de Ingeniería de Alimentos e Ingeniería Química los de mayor porcentaje. Si se comparan estas cifras con las obtenidas en las demás asignaturas del ciclo básico, se puede ver que están entre los más bajos, especialmente en programas como Ingeniería de Sistemas.

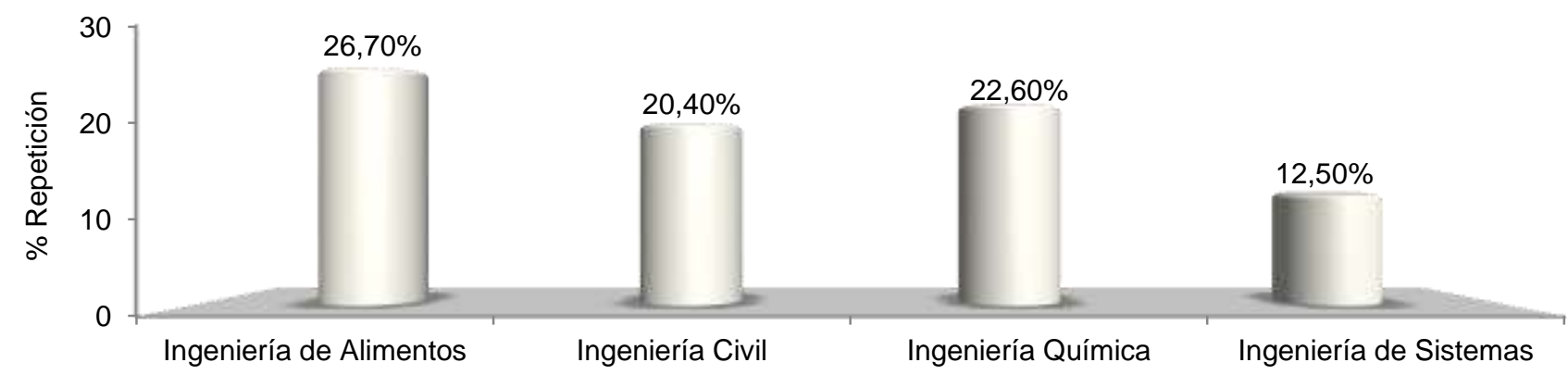

Fig. 3: Porcentaje de repetición en el área de Química en la Facultad de Ingeniería durante segundo periodo académico del 2013

Los altos niveles de repetición en las asignaturas del ciclo básico, refleja la poca preparación académica de los estudiantes en estas asignaturas. Lo cual es preocupante si se tiene en cuenta que estas son áreas de conocimiento indispensables para el desarrollo de los futuros profesionales en Ingeniería. En Colombia el promedio de repetición en el ciclo básico para las ingenierías oscila de 25,37 a 45,52\% (Cortés et al., 2011).

Los resultados de este trabajo fueron similares a los reportados por Quintero et al., (2010) quien en la Universidad Francisco José de Caldas en Colombia, encontró que las asignaturas con mayor repetición en las carreras Ingeniería Eléctrica, Electrónica, Sistemas, Industrial y Catastral, fueron en su orden, álgebra lineal $(59,33 \%)$, física mecánica $(54,68 \%)$, cálculo diferencial $(54,66 \%)$, cálculo integral $(46,66 \%)$, ecuaciones diferenciales (43,16\%) y cálculo vectorial (37,51\%). Así mismo, Rodríguez, (2013) en la Universidad Nacional de Colombia, encontró que la Facultad de Ingeniería fue la de mayor repetición, y las 
asignaturas con más incidencia fueron, matemáticas básicas $(57,35 \%)$, cálculo diferencial $(39,45 \%)$, física mecánica $(38,46 \%)$, cálculo integral $(37,82 \%)$, física eléctrica $(37,56 \%)$ y química básica $(31,56 \%)$. Lo cual el autor atribuyó a las falencias académicas de los alumnos durante su formación secundaria.

En la Tabla 3, se muestra las principales causas que originaron la repetición al interior de la Facultad de Ingeniería de la Universidad de Cartagena. No se encontraron diferencias significativas entre los porcentajes obtenidos en hombres y mujeres $[F=1,10$ y $(p=0,44>0,05)]$. Lo que indica que las causas de la repetición fueron las mismas indistintamente del género. Se puede ver que la mayoría $(41,3 \%)$, indicó que su repetición fue por los malos hábitos, indisciplina y poco tiempo dedicado al estudio, así como por la inadaptabilidad al autoaprendizaje.

Tabla 3: Principales causas de la repetición en la Facultad de Ingenierías Universidad de Cartagena

\begin{tabular}{|l|c|c|c|}
\hline Parte (A) del cuestionario - Causas de la repetición académica & $\begin{array}{c}\text { Hombres } \\
(\mathrm{n}=40)\end{array}$ & $\begin{array}{c}\text { Mujeres } \\
(\mathrm{n}=40)\end{array}$ & $\begin{array}{c}\text { Total } \\
(\mathrm{n}=80)\end{array}$ \\
\hline Malos hábitos, indisciplina y poco tiempo de dedicación al estudio. & 27.5 & 22.5 & 25.0 \\
\hline No están adaptados a la metodología de autoaprendizaje en la Universidad. & 15.0 & 17.5 & 16.3 \\
\hline No tienen lugares adecuados para realizar actividades académicas en sus casas & 10.0 & 15.0 & 12.5 \\
\hline Falta de motivación para estudiar, y Dificultades con los temas & 10.0 & 15.0 & 12.5 \\
\hline No tienen implementos para el desarrollo académico como: computadores, libros. & 5.0 & 10.0 & 7.5 \\
\hline No han tenido una preparación muy rigurosa desde la secundaria. & 5.0 & 7.5 & 6.3 \\
\hline No asistencia a clase y la asignatura no llena sus expectativas. & 7.5 & 2.5 & 5.0 \\
\hline No le gusta la forma de enseñar del docente e Incompatibilidad con compañeros & 6.0 & 4.0 & 5.0 \\
\hline Se les presentó un inconveniente al momento de realizar un examen final. & 5.0 & 5.0 & 5.0 \\
\hline No tienen tutores que los guíen en las asignaturas del ciclo básico. & 5.0 & 5.0 & 5.0 \\
\hline Total & $100 \%$ & $100 \%$ & $100 \%$ \\
\hline
\end{tabular}

Es importante resaltar que gran parte de los alumnos (12,5\%), afirmaron no tener un espacio adecuado en sus casas para realizar las actividades académicas, lo que posiblemente puede ser atribuido a las difíciles condiciones económicas de los jóvenes. Al analizar las hojas de vida de los alumnos por programa, se constató que cerca del $85,2 \%$ de Ingeniería de Alimentos, el $69,8 \%$ de Ingeniería de Sistemas, el $54,3 \%$ de Ingeniería Civil y el 50,6\% de Ingeniería Química, provienen de estratos socioeconómicos (1, 2 y 3), con familias numerosas y de municipios aledaños a la ciudad con ambientes de rezago social. Con relación a las causas de la repetición en ciencias básicas, Baquerizo et al., (2014) informó que las condiciones económicos y las carencias de conocimientos en los primeros semestres, son los principales factores que influyen en la repitencia.

Según Cortés et al., (2011) para las Facultades de Ingeniería, la mayor concentración de repetición se registra en los primeros semestres, razón por la cual se hace necesario no sólo reforzar la formación en ciencias desde el colegio, sino además mejorar los sistemas de acompañamiento estudiantil. Por su parte autores como Quintero et al., (2010) y Cerpa et al., (2014) encontraron que los altos índices de repetición en las Facultades de Ingenierías pueden ser debido a unos planes curriculares mal diseñados y a problemas con las metodologías de enseñanza-aprendizaje. Oliver et al., (2011) afirmaron que la repetición es producto de una falta de articulación entre los niveles de secundaria y universitario, especialmente en áreas de conocimiento como las de Ingeniería. Garbanzo-Vargas et al., (2007) aseguran que la falta de organización de los alumnos es un factor que puede llevar a la repetición. Ferrer et al., (2011) indica que la "libertad" que los estudiantes de primer año en Ingeniería sienten, al no estar bajo el control directo en la revisión y seguimiento de sus actividades académicas, es mal utilizada. En general tener hábitos de estudio, ser sistemático y perseverante es vital para rendir bien y no caer en repetición (Oliver et al., 2011, Carvajal et al., 2011; Tejada et al., 2013; Ramírez et al., 2013).

En la Tabla 4, se detallan los resultados generales obtenidos de las encuestas realizadas a los alumnos de los últimos semestres, la cual se hizo con el fin de identificar las principales causas del retraso académico en los programas de la Facultad de Ingenierías de la Universidad de Cartagena. Se puede ver que un gran porcentaje $(48,8 \%)$ indicó que su retraso académico fue debido a factores como: no haber terminado el trabajo de grado y a una elevada repetición durante los primeros semestres. Se resalta que en los hombres un gran porcentaje $(32,5 \%)$, indicó que su retraso académico fue debido a no tener definida su situación militar. Si se detectaron diferencias estadísticas entre hombres y mujeres en cuanto a las causas de retraso académico $[F=5,0$ y $(p=0,03<0,05)]$. Por lo que se infiere que dichas causas varían según el género. Carvajal et al., (2011) y Santamaría y Bustos, (2013) afirman que el retraso académico acarrea consecuencias como gastos de funcionamiento para las universidades, así como los gastos propios de sostenimiento para el estudiante; y que esto se refleja en una disminución de la cobertura en educación superior. 
Tabla 4: Principales causas del retraso académico en la Facultad de Ingeniería Universidad de Cartagena

\begin{tabular}{|c|c|c|c|}
\hline Parte (B) del cuestionario - Causas del retraso académico & $\begin{array}{c}\text { Hombres } \\
(n=40)\end{array}$ & $\begin{array}{c}\text { Mujeres } \\
(n=40)\end{array}$ & $\begin{array}{c}\text { Porcentaje } \\
(\mathrm{n}=80)\end{array}$ \\
\hline No han terminado el trabajo de grado & 35,0 & 20,0 & 27,5 \\
\hline No han terminado académicamente por su elevada repetición en el ciclo básico & 17,5 & 25,0 & 21,3 \\
\hline Los hombres no han definido su situación Militar & 32,5 & 0,0 & 16,3 \\
\hline No han realizado las practicas Industriales & 5,0 & 20,0 & 12,5 \\
\hline No han aprobado examen de suficiencia de Ingles & 2,5 & 17,5 & 10,0 \\
\hline No han realizado las pruebas de estado Saber Pro & 2,5 & 5,0 & 3,8 \\
\hline Consiguen trabajo y dan prioridad a otros aspectos personales & 0,0 & 7,5 & 3,8 \\
\hline Poseen deudas financieras con la Universidad & 5,0 & 0,0 & 2,5 \\
\hline Estuvo embarazada y aplazó un semestre & 0,0 & 2,5 & 1,3 \\
\hline Tienen cargos de representación estudiantil & 0,0 & 2,5 & 1,3 \\
\hline Total & $100 \%$ & $100 \%$ & $100 \%$ \\
\hline
\end{tabular}

\section{Pruebas Saber Pro de la Facultad de Ingeniería de la Universidad de Cartagena}

Son exámenes de conocimientos que presentan todos los alumnos universitarios en Colombia, próximos a culminar su formación profesional, y son obligatorias como requisito de grado (ICFES, 2014). En la Tabla 5 , se pueden observar los resultados obtenidos por los programas de la Facultad de Ingeniería de la Universidad de Cartagena, en cuanto a las competencias genéricas de las pruebas Saber Pro. Los datos representan el promedio durante los años 2010, 2011, 2012 y 2013, para cada competencia.

Tabla 5: Pruebas Saber Pro, Facultad de Ingenierías en los años 2010, 2011, 2012 y 2013

\begin{tabular}{|l|c|c|c|c|c|c|}
\hline Competencia & Referencia & \multicolumn{4}{|c|}{ Programas de la Facultad de Ingeniería } & \multicolumn{2}{c|}{ Análisis de Varianza } \\
\cline { 3 - 7 } & Colombia & Alimentos & Sistemas & Civil & Química & Valor $F$ y $p$-valor \\
\hline Ciudadanas & 10,12 & $9,94 \pm 0,03 a$ & $10,24 \pm 0,26 a b$ & $10,37 \pm 0,20 a b$ & $10,52 \pm 0,28 b$ & {$[F(4,47)$ y $(p=0,025<0,05)]$} \\
\hline Escritura & 10,19 & $9,84 \pm 0,05 a$ & $10,15 \pm 0,10 a$ & $10,24 \pm 0,21 b$ & $10,29 \pm 0,26 b$ & {$[F(8,58)$ y $(p=0,003<0,05)]$} \\
\hline Lectura crítica & 10,17 & $10,04 \pm 0,10 a$ & $10,50 \pm 0,39 a$ & $10,62 \pm 0,15 a$ & $10,46 \pm 0,32 a$ & {$[F(2,20)$ y $(p=0,141>0,05)]$} \\
\hline Razonamiento & 10,57 & $10,02 \pm 0,04 a$ & $10,61 \pm 0,45 a b$ & $11,05 \pm 0,24 b$ & $11,02 \pm 0,09 b$ & {$[F(6,27)$ y $(p=0,009<0,05)]$} \\
\hline
\end{tabular}

El análisis de varianza para los datos de la competencia ciudadanas entre los programas, indicó diferencias $(p<0,05)$, al aplicar prueba de Scheffé, se observó que entre los programas Sistemas y Civil no hubo diferencias (letras iguales en una misma fila), mientras que en Ingeniería de Alimentos e Ingeniería Química si hubo diferencias estadísticamente significativas (letras diferentes en una misma fila). Mientras que los datos de lectura crítica fueron estadísticamente iguales $(p>0,05)$. Todos estos valores se contrastaron con la referencia para las Ingenierías en Colombia. Se observó ver que el promedio de escritura entre los cuatro programas fue de $(10,13 \pm 0,20)$, y no superó el mínimo de referencia para Ingeniería en Colombia. En general el programa Ingeniería de Alimentos obtuvo los resultados más bajos en todas las competencias evaluadas, por lo que se sugiere mayor atención en estos aspectos formativos. En la Tabla 6, se esquematizan los resultados promedios de las evaluaciones en inglés, de los años 2010, 2011, 2012 y 2013. Estos datos representan los porcentajes de estudiantes en los respectivos niveles por programa durante el periodo analizado.

Tabla 6: Pruebas Saber Pro de Ingles para la Facultad de Ingenierías de los años 2010, 2011, 2012 y 2013

\begin{tabular}{|c|c|c|c|c|}
\hline \multirow{2}{*}{ Niveles de Inglés } & \multicolumn{4}{|c|}{ Programas de la Facultad Ingeniería Universidad de Cartagena } \\
\cline { 2 - 5 } & Alimentos & Civil & Sistemas & Química \\
\hline A & 16,60 & 16,50 & 0,90 & 0 \\
\hline A1 & 44,60 & 18,40 & 7,60 & 3,40 \\
\hline A2 & 18,10 & 25,30 & 27,60 & 18,60 \\
\hline B1 & 13,20 & 21,20 & 17,20 & 38,20 \\
\hline B2 & 7,50 & 18,60 & 46,70 & 39,80 \\
\hline Total & $100 \%$ & $100, \%$ & $100 \%$ & $100 \%$ \\
\hline
\end{tabular}

Se puede ver que al sumar los niveles más bajos A, A1, A2, el programa Ingeniería de Alimentos obtuvo un $79,3 \%$; seguido por Ingeniería Civil con un $60,2 \%$, lo que indica la poca preparación en segunda lengua de los alumnos egresados de estos programas. Lo que debe ser tenido en cuenta en busca de mejorar, ya que cada día la sociedad exige ingenieros que tengan habilidades comunicativas, especialmente con el manejo y dominio del inglés como segundo idioma (Barragán et al., 2011). Los niveles más altos, estuvieron en Ingeniería Química e Ingeniería de Sistemas con un 78,1\% y 63,9\% de sus alumnos en los niveles B1 y B2, respectivamente, lo que muestra un mayor desarrollo de sus egresados en segundo idioma. 


\section{CONCLUSIONES}

Los alumnos presentaron altos niveles de repetición en las asignaturas del ciclo básico, especialmente en las áreas de física, y cálculo. El 48,8\% de los alumnos indicó que su repetición se debió a, malos hábitos de estudio, inadaptabilidad al autoaprendizaje, desinterés por las temáticas y falta de preparación académica. No se presentaron diferencias entre hombres y mujeres, lo que indica que las causas de la repetición fueron las mismas indistintamente del género. Las causas del retraso académico fueron en su mayoría académicas, principalmente no haber terminado el trabajo de grado, lo cual reveló que a los alumnos de los últimos semestres no se les brindó opciones diferentes al trabajo de grado, para alcanzar su título profesional. Se detectaron diferencias entre hombres y mujeres, por lo que se infiere que las causas del retraso académico varían según en género. Es importante buscar alternativas que ayuden a reducir la repetición y retraso académico en la Facultad de Ingeniería de la Universidad de Cartagena.

\section{REFERENCIAS}

Baquerizo, R., O. Amechazurra y J. Galarza. La repetición en las instituciones de Educación Superior: algunas experiencias investigativas en el Ecuador." Universidad y Sociedad, Revista Científica de la Universidad de Cienfuegos: 6 (1) 102-107 (2014).

Barragán M. La carga horaria excesiva, factor del bajo rendimiento en Ingeniería Civil: Un estudio de Caso, Revista Tecnología, Investigación y Docencia, 6, 67-73 (2011).

Carvajal, P., A. A. Trejos y M. Barrera, Factors associated with the permanence of school desertion in the Department of Risaralda 2010, Scientia et Technica: 16 (48) 71-76 (2011).

Cerpa, F., P. González y S. Cantillo. Análisis comparativo entre las características más relevantes de deserción estudiantil en el programa de Ingeniería Industrial de la Universidad Autónoma del Caribe, Escenarios: 12(1) 96-104, (2014).

Cortés, H., L. Gallego y G. Rodríguez, La Facultad de Ingeniería hoy: una aproximación hacia la construcción de indicadores académicos, Ingeniería e Investigación, 31(1) 74-90 (2011).

Ferrer, K. y otros cinco autores, Student achievement in analytical chemistry: a comparative study on two syllabi, Investigación Arbitrada: 15 (52) 651-662 (2011).

Garbanzo-Vargas, Factores asociados al rendimiento académico en alumnos universitarios, una reflexión desde la calidad de la educación superior pública, Revista Educación: 31(1) 43-63, (2007).

González, L. Estudio sobre la repetición y deserción en la educación superior Chilena. Digital Observatory for higher education in Latin America and The Caribbean, IESALC-UNESCO, Santiago-Chile (2005).

ICFES. Instituto Colombiano para el fomento de la Educación Superior. Resultados por programa académico Examen de Estado de Calidad de la Educación Superior, 2014. Disponible en URL: http://www.icfesinteractivo.gov.co/resultadosSaberPro/Acceso 7 de agosto, (2014).

Oliver M.C, y otros tres autores, Attendance and desertion in general chemistry at the engineering courses of the national technological university - Córdoba regional faculty (UTN-FRC), Argentina, Avances en Ciencias e Ingeniería: 2(2) 117-129 (2011).

Quintero, R. y otros cuatro autores. Informe sobre prueba académica y deserción estudiantil en la Universidad Distrital Francisco José de Caldas (2010).

http://virtual.udistrital.edu.co/licquimica/files/Informe.pdf. Acceso 5 de agosto, (2014).

Ramírez, P. y C. Fuentes, Felicidad y Rendimiento Académico: Efecto Moderador de la Felicidad sobre Indicadores de Selección y Rendimiento Académico de Alumnos de Ingeniería Comercial, Formación

Universitaria: 6(3), 21-30 (2013).

Rodríguez G. Pérdida de asignaturas en la Universidad Nacional de Colombia, (2013). http://www.bogota.unal.edu.co/otros/noviembre15/Perdidas_2013-I.pdf. Acceso 5 de agosto, (2014).

SACES - Sistema de Aseguramiento de la Calidad de Educación Superior. (2014) Disponible en URL: http://www.mineducacion.gov.co/Sistemasdeinformacion/1735/property.html. Acceso 7 de agosto (2014).

Santamaría, F. y A. Bustos, Permanence and Dropout Rates in Higher Education: A Research Experience Based on Young Students Voices, Revista Infancias Imágenes: 12 (2) 73-80 (2013)

Soria-Barreto K. y S. Zúñiga-Jara, Aspectos Determinantes del Éxito Académico de Estudiantes Universitarios, Formación Universitaria: 7(5) 41-50 (2014)

Tejada, C., A. Villabona y E. Ruiz. Deserción y Repetición del Programa de Ingeniería Química de la Universidad de Cartagena Periodo 2006-2011, Revista ciencia e Ingeniería al Día: 8 (1) 55-66 (2013). 